\title{
Assessing the Quality of Pharmacy Care: It's Not About Adherence
}

\author{
Michael T. Rupp, BSPharm, PhD, FAPhA
}

Rupp's Viewpoint

$\mathrm{I}$ will concede that adherence to prescribed medication therapy is generally a good thing and should usually be encouraged by pharmacists during their interactions with patients. I say "usually" because pharmacists also have an important and underappreciated role in discouraging the unnecessary and inappropriate use of prescription drugs through drug therapy monitoring and deprescribing activities. ${ }^{1,2}$

I will also allow that pharmacists in institutional practice settings who are in positions to directly monitor and influence medication administration have at least a shared responsibility to ensure that patients are receiving their medications as prescribed. This oversight responsibility is typically shared with nursing staff and uses the comparably reliable data source of medication administration records to monitor adherence.

That said, I reject the assertion that medication adherence is a valid indicator of the quality of care provided by community pharmacies or pharmacists for 3 reasons.

First, the most commonly used method for measuring medication adherence, proportion of days covered (PDC) does not actually measure adherence (see calculation below).

$$
\begin{aligned}
& \text { Proportion of } \\
& \text { Days Covered }
\end{aligned}=\frac{\begin{array}{l}
\text { (Number of days with } \\
\text { medication on hand) }
\end{array}}{\begin{array}{l}
\text { (Number of days in } \\
\text { observation period) }
\end{array}} \times 100 \%
$$

Rather, this metric merely provides an estimate of maximum potential adherence as inferred from prescription refill data. All we really know from a PDC value is that a particular number of medication doses have been dispensed. Beyond that, everything else is an assumption, an unreliable and clinically risky practice upon which I recently commented. ${ }^{3}$ The mere fact that a patient is obtaining routine refills does not necessarily mean the patient is adhering to the prescribed medication regimen. Similarly, inconsistent refills do not necessarily indicate poor adherence, since patients often have alternative sources for their medications.

Beyond equivocal interpretation, as Canfield et al. describe in this issue of $J M C P,{ }^{4}$ there are also methodological challenges

J Manag Care Spec Pharm. 2019;25(10):1046-47

Copyright $\odot 2019$, Academy of Managed Care Pharmacy. All rights reserved. that must still be overcome if we are to calculate PDC in a sufficiently accurate and consistent manner to support uniform and unambiguous interpretation and use, as they succinctly summarize in Figure 2 of their article. Something as seemingly unequivocal as the medication fill date can have multiple meanings across different pharmacy systems and/or data analysts.

Second, the standard acceptable PDC value of $80 \%$ further assumes that a patient (any patient) who takes $80 \%$ of their prescribed medication (any medication) is sufficiently adherent to ensure adequate control of their medical condition (any condition). Even if our measure of adherence were accurate, this assumption simply does not square with pharmacological therapeutics. There are probably patients and medications for which less than $80 \%$ adherence is sufficient, and there are certainly patients and medications for which $80 \%$ adherence to their prescribed therapy is dangerously inadequate. Thus, if a measure of medication adherence were to be useful, the targeted performance standard must be individualized to the medication, the patient, and the condition being treated.

My third and most important objection, is that even if it were measured accurately, and targets were tailored to individual patients, medication adherence would still not be a valid measure of the quality of care provided by a pharmacy or pharmacist. While adherence is frequently referred to as a health outcome, it is clearly not. It fails to meet the criteria to be considered a legitimate metric for assessing the quality of pharmacy or pharmacist care from the perspective of either structure, process, or outcome. ${ }^{5}$ At best, medication adherence is an intermediate or surrogate outcome that may be correlated—with indeterminate reliability—with some clinical outcomes we seek to achieve with medication therapy.

It should be noted, however, that adherence actually is an ultimate desired outcome of pharmaceutical manufacturers, wholesalers, retailers, and other vested interests in the channel of distribution for prescription drugs because it correlates very highly with revenue. However, there is an important distinction between maximizing medication consumption and optimizing medication adherence. From a quality perspective, pharmacists should be focused exclusively on the latter. One has to wonder if those interests are at least partly responsible for the relentless effort in recent years to make medication adherence Job \#1 for retail pharmacists, as opposed to activities that really are direct indicators of the quality of care they 
provide, such as dispensing accuracy, prospective drug utilization review, and patient counseling.

So, if PDC has such severe limitations, why is it so widely used? The primary reason is because prescription claims data are readily available. It has always reminded me of the story of a woman who finds her husband searching the living room of their house:

Wife: "What are you looking for?"

Husband: "My cufflink."

Wife: "Where did you lose it?"

Husband: "In the bedroom."

Wife: "Why are you looking for it here?"

Husband: "Because the light is better."

Such a rationale is not a good justification for a metric that purports to assess the quality of care provided by pharmacists and the organizations within which they practice. It is particularly distressing that this flawed metric has gained such prominence in the assessment of direct and indirect remuneration fees that can dramatically affect pharmacy payment in many health plans or even limit the ability of a pharmacy to participate in some plans.

I agree with Dr. Campbell that the quality of pharmacist care is a multidimensional construct that requires a multifaceted approach to represent adequately. I also agree that using any single metric to assess the quality of pharmacist care would be analogous to assessing vehicle safety based exclusively on how well the brakes work. The difference is that functioning brakes really are a legitimate indicator of a vehicle's safety.

So then, what is the appropriate interpretation and use of medication adherence, assuming that we were able to measure it accurately? Ultimately, adherence is merely a measure of patient behavior that typically results from conscious, voluntary decisions by patients. Returning to Dr. Campbell's example, using medication adherence as an indicator of the quality of pharmacist care is therefore analogous to using someone's driving behavior as an indicator of the mechanical safety of the vehicle the individual is driving.

But could an accurate measure of adherence assist a pharmacist to provide better care? Absolutely. Such a metric could alert the pharmacist that a patient may require additional counseling to understand their therapy and/or assistance to overcome one of the many known barriers to adherence. The final decision of whether and how to comply with a prescribed medication regimen, however, is exclusively the patient's to make. Moreover, that decision is not a reliable indicator of the quality of care provided by the pharmacist any more than it would be for the clinician who prescribed the medication.

In the final analysis, the pharmacist's legal, professional, and ethical responsibility is to ensure that the patient has adequate knowledge and resources to make and implement a fully informed decision regarding adherence to their medication therapy. The rest is up to the patient.

As for Dr. Campbell's recommendation that "a multistakeholder, consensus-based, transparent method should be used to develop a core set of pharmacy/pharmacist quality measures," I could not agree more. My question is, why has this still not been done?

\section{Authors}

MICHAEL T. RUPP, BSPharm, PhD, FAPhA, Midwestern University College of Pharmacy, Glendale, Arizona.

AUTHOR CORRESPONDENCE: Michael T. Rupp, BSPharm, PhD, FAPhA, Midwestern University College of Pharmacy, 19555 N. 59th Ave., Glendale, AZ 85383. Tel.: 623.572.3528;

E-mail: MTRUPP@midwestern.edu.

\section{DISCLOSURES}

No funding supported the writing of this article. The author has nothing to disclose.

\section{REFERENCES}

1. D'Arrigo T. Deprescribing is the cure for 'disease' of polypharmacy. Pharmacy Today. December 2018. Available at: https://www.pharmacytoday. org/article/S1042-0991(18)31658-X/pdf. Accessed August 31, 2019.

2. Whitman A, DeGregory K, Morris A, Mohile S, Ramsdale E. Pharmacistled medication assessment and deprescribing intervention for older adults with cancer and polypharmacy: a pilot study. Support Care Cancer. 2018;26(12):4105-13. Available at: https://www.ncbi.nlm.nih.gov/pmc/ articles/PMC6204077/. Accessed August 31, 2019.

3. Rupp MT. Encouraging students to challenge assumptions. Am J Pharm Educ. 2019;83(5):7481. Available at: https://www.ajpe.org/doi/abs/10.5688/ ajpe7481. Accessed August 31, 2019.

4. Canfield SL, Zuckerman A, Anguiano RH, Jolly JA, DeClercq J. Navigating the wild west of medication adherence reporting in specialty pharmacy. J Manag Care Spec Pharm. 2019;25(10):1073-77.

5. Rupp MT. Assessing quality of care in pharmacy: remembering donabedian. J Manag Care Spec Pharm. 2018;24(4):354-56. Available at: https://www. jmcp.org/doi/10.18553/jmcp.2018.24.4.354. 